\title{
MODEL-BASED 3-D RECONSTRUCTION OF BRANCHING VESSELS
}

\author{
Jeff Fessler, Dwight Nishimura, and Albert Macovski
}

\author{
Information Systems Laboratory
}

Stanford University

\section{ABSTRACT}

This paper describes a new approach to the problem of reconstructing a 3-D arterial tree model from a few angiographic projections. We first develop a natural optimality criterion that defines the globally best reconstruction, and then outline an algorithm that maximizes this criterion. The criterion is a compromise between the conflicting goals of measurement likelihood and object smoothness. Our approach uses a parametric model for the arterial tree, with a new, more accurate model for bifurcations. The algorithm is demonstrated on simulated projections and on magnetic resonance (MR) images.

\section{INTRODUCTION}

Accurate 3-D reconstructions of arterial trees would be useful tools for surgical planning and quantitative diagnosis. However, current angiographic methods (both X-ray and MR) only provide a few simultaneous projections. Given only a few projections, the inverse problem of reconstructing the 3-D objects that generated those projections is severely underdetermined. Fortunately, one can overcome this problem by utilizing a priori knowledge about the structure of blood vessels.

A few vessel reconstruction algorithms have recently been presented that are based on a generalized cylinder (GC) object model with elliptical cross sections $[1,2]$. These approaches have not accurately reconstructed branching vessels. We describe below an extended object model that includes cross sections consisting of two overlapping ellipses. This model is more accurate than a single ellipse for bifurcations, where important pathologies frequently occur. Under this model, the reconstruction problem is equivalent to the problem of es timating the ellipse parameters using the measured projections.

It is difficult to statistically analyze the performance vessel reconstruction algorithms that are not based on an optimality criterion. We describe one such criterion below, along with its mathematical and physical motivations. Then we outline an algorithm for computing the optimal estimate under that criterion. The result is a parametrically reconstructed arterial tree that can be displayed for surgical planning or analyzed for quantitative diagnosis.

\section{MEASUREMENT MODEL}

If we knew the 3-D object (arterial tree) distribution, we could compute its ideal projections using the Radon transform. Let $P_{\theta}(\cdot)$ denote the vector of 2-D samples of the projection function at angle $\theta . P_{\theta}$ is determined by measurement geometry, detector pixel size, and point spread function. Let $Y_{\theta_{m}}$ denote an actual measured projection. We are only given $Y_{\theta}$ for a few values of $\theta$; one reasonable set is dual bi-plane projections [2], where $\theta_{m} \in\{0, \phi, 90,90+\phi\}$ for some small $\phi$. Let $Y=\left[Y_{\theta_{1}}, \ldots, Y_{\theta_{M}}\right]$ be the aggregate measurement vector and similarly define $P$. Since the measurements are corrupted by noise, we use the measurement model:

$$
Y=P(\text { Object })+N,
$$

where $N$ is assumed Gaussian. This model is exact for MR angiography, and is approximate for X-ray angiography.

Naturally we would like the projections of the reconstructed object to be close to the measured projections. This leads to the measurement likelihood:

$$
L_{M}(\text { Object })=\| Y-P(\text { Object }) \|^{2} .
$$

A locally optimal approach to reconstruction is to find the object that minimizes $L_{M}$. This approach ignores the fact that blood vessels are fairly smooth, i.e., the parameters of the elliptical cross section vary slowly along the vessel axis. Before quantifying this smoothness, we first describe the GC model for a single bifurcating object.
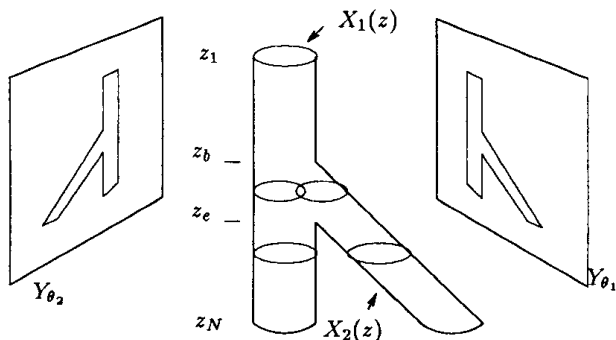

Figure 1: Projections of a bifurcation. The problem is to reconstruct $X_{1}(z)$ and $X_{2}(z)$ from the $Y$ 's.

\section{OBJECT MODEL}

For simplicity, we consider the single-bifurcation object shown in Fig. 1. Let the projections be acquired around a rotation axis roughly parallel to the axis of the vessels, and let $z$ denote the axial coordinate. The vessel cross sections are modeled as ellipses with six parameters: position $(x, y)$, radius, eccentricity, orientation, and density (contrast). For better accuracy in the vicinity of the bifurcation, we model the cross section as two overlapping ellipses with the same density. The branch object of Fig. 1 is thus completely specified by the following parameters:

$$
B=\left\{z_{b}, z_{\varepsilon},\left\{X_{1}(z)\right\}_{z=z_{1}}^{z_{N}},\left\{X_{2}(z)\right\}_{z=z_{b}}^{z_{N}}\right\},
$$

where $X_{1}(z)$ and $X_{2}(z)$ are the ellipse parameters of the cross sections of the principal and secondary vessels respectively. Since a single ellipse and two disjoint ellipses are special cases of two overlapping ellipses, $z_{b}$ and $z_{e}$ do not need to be known exactly.

Before we quantify the object smoothness, consider the following: if a thin rod is stretched along a set of pins at coordinates $\left\{\left(x_{i}, y_{i}\right)\right\}_{i=1}^{N}$, then the curve formed by the rod will minimize its tension: $\int \ddot{y}^{2}(x) d x$. The resulting curve is an interpolating cubic spline: the smoothest 
curve through the given points. Similarly, it is reasonable to assume that arteries take the smoothest path to their destination, subject to certain anatomical constraints. Likewise, to maximize fluid pressure, the vessel cross section will change slowly. This motivates our definition of object likelihood:

$$
L_{O}(B)=\alpha_{1}^{T} \int_{1}^{N} \ddot{X}_{1}^{2}(z) d z+\alpha_{2}^{T} \int_{z_{b}}^{N} \ddot{X}_{2}^{2}(z) d z .
$$

This non-parametric approach is closely related to spline smoothing [3]. The constants $\alpha_{i}$ control the penalty for object roughness. A unique feature of the algorithm given below is that these constants are estimated from the data itself. Thus the algorithm will adapt to vessel abnormalities such as stenoses (narrowings). (2) easily extends to measure the likelihood of an arterial tree.

\section{RECONSTRUCTION ALGORITHM}

We would like to find an object minimizing both $L_{M}$ and $L_{O}$, but these goals conflict in general. Thus, we use the "penalized likelihood" [3] compromise as our optimality criterion:

$$
\hat{B}=\arg \min _{B} L_{M}(B)+L_{O}(B) .
$$

Having explicitly defined this criterion, we could now use statistical tools to analyze the estimation accuracy, and to generate confidence intervals for the estimates [3]. Here, we develop an efficient algorithm for performing the minimization (3).

First find the maximum measurement likelihood estimate:

$$
B_{0}=\arg \min _{B} L_{M}(B) \text {. }
$$

Using the first order Taylor's expansion:

$$
P(B) \approx P\left(B_{0}\right)+J_{0}\left(B-B_{0}\right)
$$

where $J_{0}$ is the Jacobian of $P$ at $B_{0}$, we can rewrite (3):

$$
\hat{B}=\arg \min _{B}\left\|\left(Y-P\left(B_{0}\right)+J_{0} B_{0}\right)-J_{0} B\right\|^{2}+L_{O}(B) .
$$

After some manipulations, this can be written:

$$
\hat{B}=\arg \min _{B}\left\|\left(J_{0}^{\prime} J_{0}\right)^{-\frac{1}{2}}\left(B_{0}-B\right)\right\|^{2}+L_{O}(B) .
$$

This final form is a special case of the multi-dimensional generalization of cubic-spline smoothing. (6) is efficiently evaluated using the procedure described in [4]. The smoothing parameters $\alpha_{i}(2)$ are automatically estimated from the data using cross-validation [4]

\section{RESULTS}

We applied the algorithm to synthetic projections $(\theta=0,30,90$, 120 ) of a branching object with an eccentric stenosis. Despite severe noise, the estimation error is well below a pixel, as can be seen in Fig. 2. We also applied the algorithm to MR angiograms $[5](\theta=0,45,90,135)$ of a flow phantom. Structured noise in the background of the MR projections has introduced some errors into the reconstruction. These errors will decrease as the MR angiography techniques improve. Relinearizing (4) about the estimated object and repeating the estimation (5) could also improve the accuracy.

\section{CONCLUSIONS}

We have defined a natural optimality criterion for global reconstruction of an arterial tree from its projections, including accurate modeling and reconstruction of bifurcations. We have derived an algorithm that efficiently computes the best estimate, and have demonstrated the viability of the approach on simulated projections and MR images. We will next evaluate the algorithm on angiograms of human carotid arteries.
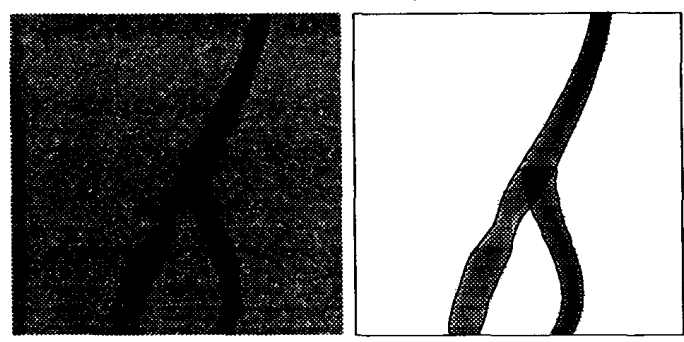

Figure 2: Left: One of four simulated projections; $\theta=0^{\circ}$. Right Outline of reprojection of reconstructed object (black) superimposed on true object (shaded) at $\theta=0^{\circ}$.
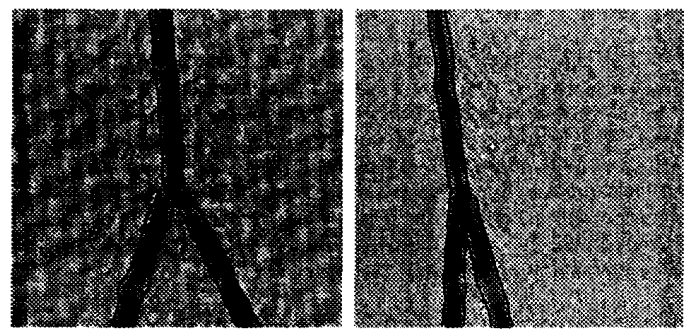

Figure 3: Outline of reprojection of reconstructed object (black) superimposed on original MR projections of tube phantom (shaded) left: $\theta=90^{\circ}$, right: $\theta=0^{\circ}$.

\section{REFERENCES}

[1] K. Kitamura, J. Tobis, and J. Sklansky, "Estimating the 3-D skeletons and transverse areas of coronary arteries from biplane angiograms," IEEE Transactions on Medical Imaging, vol. 7, pp. 173187, Sep. 1988.

[2] J. Fessler and A. Macovski, "3-D reconstruction of vessels with stenoses and aneurysms from dual bi-plane angiograms," in Proceedings, SPIE Medical Imaging III Conference, Feb. 1989.

[3] B. Silverman, "Some aspects of the spline smoothing approach to non-parametric regression curve fitting," Journal of the Royal Statistical Society Series $B$, vol. 47, pp. 1-52, 1985.

[4] J. Fessler and A. Macovski, "Non-parametric fixed-interval smoothing with cubic splines," 1989 . Submitted to IEEE Trans. on Acoustics, Speech, and Signal Proc.

[5] D. Nishimura, A. Macovski et. al., "Magnetic resonance angiography by selective inversion recovery using a compact gradient echo sequence," Magnetic Resonance in Medicine, vol. 8, pp. 96-103, 1988 .

102 Durand

Information Systems Laboratory

Stanford, CA 94305

(415)-723-1904/3311

ARPA: fessler@isl.stanford.edu 